\title{
Ability of Therapists Trained in Mechanical Diagnosis and Therapy to Guess Pain Catastrophizing and Kinesiophobia Scores for Patients with Low Back Pain
}

\author{
Katsuya Suzuki, Hiroshi Takasaki ${ }^{*}$ \\ Department of Physical Therapy, Saitama Prefectural University, Koshigaya, Saitama, Japan \\ Email: *physical.therapy.takasaki@gmail.com
}

How to cite this paper: Suzuki, K. and Takasaki, H. (2020) Ability of Therapists Trained in Mechanical Diagnosis and Therapy to Guess Pain Catastrophizing and Kinesiophobia Scores for Patients with Low Back Pain. Open Journal of Therapy and Rehabilitation, 8, 119-130.

https://doi.org/10.4236/ojtr.2020.84011

Received: July 20, 2020

Accepted: August 25, 2020

Published: August 28, 2020

Copyright $\odot 2020$ by author(s) and Scientific Research Publishing Inc. This work is licensed under the Creative Commons Attribution International License (CC BY 4.0).

http://creativecommons.org/licenses/by/4.0/

\begin{abstract}
Objective: To investigate whether a physical therapist trained through the $\mathrm{Me}$ chanical Diagnosis and Therapy (MDT) diploma program could guess psychological Patient Reported Outcome Measure (PROM) scores of individuals with low back pain (LBP) by taking patient history and completing a physical evaluation. Methods: Ten participants with LBP completed PROMs immediately before history taking and again after a physical evaluation. PROMs included the Pain Catastrophizing Scale (PCS), Tampa Scale for Kinesiophobia (TSK), Hospital Anxiety and Depression Scale, and Pain Coping Strategy Questionnaire. A physical therapist who completed the MDT diploma program took the patients' history and completed their physical evaluation. The therapist completed the same PROMs immediately after both history taking and physical evaluation. Correlations between patient and therapist scores were calculated using Spearman's $\rho$. Results: Statistically significant positive correlations were detected in the PCS $(\rho=0.65)$ and TSK $(\rho=0.78)$ before history taking, and in the PCS ( $\rho=$ $0.81)$ and TSK $(\rho=0.74)$ after physical evaluation. Discussion: The results are based on one MDT therapist and generalizability of the findings is limited. However, the current preliminary findings justify the need for further studies to explore effective post-graduate training to promote a patient centered approach.
\end{abstract}

\section{Keywords}

History Taking, McKenzie Approach, Physical Evaluation, Post-Graduate Training, Psychological Status

\section{Introduction}

Considering and appropriately addressing psychosocial barriers is a widely ac- 
cepted measure for successful management of low back pain (LBP) [1] [2] [3]. A study by Miki et al. [4] included 78 patients with LBP and 21 physical therapists, and investigated whether the physical therapists could guess the scores of psychological Patient Reported Outcome Measures (PROMs) through physical therapy evaluation. The physical therapists could not guess the psychological PROM scores and the clinical experiences of the physical therapists did not enhance the accuracy of their guess. However, another study [5] showed that trained physical therapists could guess patient anxiety through physical therapy evaluation. A potential reason for the discrepancy between the studies [4] [5] is the physical therapists' training level, and it was added to a research agenda to investigate whether certain post-graduate training programs might affect the ability of physical therapists to guess patient psychological PROM scores through physical therapy evaluation [4]. One promising post-graduate training program available around the world is the Mechanical Diagnosis and Therapy (MDT).

The MDT [6] is a system for the assessment and treatment of musculoskeletal conditions, and is the most commonly used physical therapy management for LBP [7] [8] [9] [10]. The MDT involves detailed history taking using a semi-structured form and physical evaluation utilizing patient responses to loading strategies, where empowerment of self-management and patient education are always emphasized [6]. There has been increasing evidence that the MDT is effective in enhancing patient attitudes toward self-management [11] and improving psychosocial outcomes [12]. Further, MDT therapists are more biopsychosocially-oriented than other physical therapists for LBP management [13].

There are two levels of certification in the MDT training system-credential and diploma. Briefly, the diploma level requires $240 \mathrm{~h}$ of online training, with topics including the potential drivers of pain and disability. The diploma level also requires further understanding of MDT on top of the $112 \mathrm{~h}$ of credential training. Further, the diploma training requires $360 \mathrm{~h}$ of clinical practice based on a variety of musculoskeletal disorders: for example, maximizing treatment efficiency, effectiveness by the appropriate provision of education, self-management procedures, clinician procedures, and preventative measures. The differences are explained in more depth in a previous study [14].

The primary aim of the current study was to investigate the possibility that a physical therapist trained through the MDT diploma program could guess PROM scores of individuals with LBP, which were used in the previous study [4], through history taking and physical evaluation. In this study, we conducted a pilot test with one MDT therapist. The secondary aim of the study was to investigate immediate effects of MDT on psychological status of those with LBP.

\section{Methods}

\subsection{Design}

This pilot study includes MDT evaluation, which also includes a potential inter- 
vention effect. Therefore, this study was pre-registered in a clinical trial registry (UMIN000037990). The institutional research ethics committee (Saitama Prefectural University, \#19100) approved the current study. All participants provided written consent before data collection.

\subsection{Participants}

Using convenience sampling, we recruited participants with LBP via advertising at the university (Saitama Prefectural University, Koshigaya, Saitama, Japan) between November 2019 and March 2020. The inclusion criteria were: 1) age 18 - 60 years, 2) at least one episode of LBP for at least two successive days with intensity of at least two in the 11-point numerical rating scale (NRS) during the last month [15] [16], and 3) a score on the Oswestry Disability Index (ODI) [17] [18] of $13 \%$ or more, where $12 \%$ is the cut-off score for patients with LBP disability in the Japanese population [19]. Exclusion criteria were: 1) undertaking treatment for LBP during the study period, 2) congenital disorders, 3) diagnosed neurological disorders, 4) diagnosed mental disorders, 5) being pregnant or multipara, and 6) diagnosed spinal deformity.

\subsection{Physical Therapist}

An author (HT) undertook MDT history taking and physical evaluation of participants. He had completed the MDT diploma program but had not yet taken the MDT diploma examination at the time.

\subsection{Procedures}

The participants completed the following PROMs immediately before having their MDT history taken: 1) the P4 for pain intensity, 2) the ODI for disability due to LBP, 3) the 12-item Orebro Musculoskeletal Screening Questionnaire (OMSQ) for general understanding of the risk of poor prognosis, 4) the Central Sensitization Inventory (CSI) for pain status, 5) the Pain Catastrophizing Scale (PCS) for pain catastrophizing, 6) the Tampa Scale for Kinesiophobia (TSK) for kinesiophobia, 7) the Hospital Anxiety and Depression Scale (HADS) for anxiety and depression, and 8) the two-item Pain Coping Strategy Questionnaire (PCSQ) for confidence in a management strategy for LBP. Participants' age, gender, height, weight, and symptom duration and location were also collected as demographics.

The participants also completed the following PROMs immediately after the MDT physical evaluation: 1) the PCS, 2) the TSK, 3) the HADS, 4) the PCSQ, and 5) the 11-point global recovery of change scale (GRCS) [20].

The physical therapist completed the following measures immediately after taking patient history and again immediately after the physical evaluation: 1) the PCS, 2) the TSK, 3) the HADS, and 4) the PCSQ.

The physical therapist was blinded to the patient PROMs results. The content of MDT history taking was not controlled and was tailored to each participant 
by the physical therapist, so each item of the PROMs was not necessarily covered. Each MDT session lasted $60 \mathrm{~min}$.

\subsection{Outcomes}

The primary outcomes in the correlation analysis were the same PROMs used in the previous study [4], including the PCS, the TSK, and HADS.

\subsubsection{Demographics}

Symptom duration was defined as the number of days and/or months since the last pain-free month [16]. Symptom location was assessed with the help of a body chart ( 1 = lower back, $2=$ side of the lower back, $3=$ buttock, $4=$ thigh, $5=$ lower leg, and 6 = foot), where a higher score indicated more distal pain [21].

\subsubsection{The ODI}

The ODI is a well-established PROM for disability due to LBP. We used a Japanese version of the ODI [17], where a higher score indicates greater disability due to LBP.

\subsubsection{The $P 4$}

The P4 is composed of four 11-point NRSs for pain intensity, where higher sum scores indicate higher pain intensity over the last two days [22]. The construct validity and test-retest reliability (intra-class correlation coefficient $[$ ICC] $=0.78$ ) have been established [22].

\subsubsection{The OMSQ}

We used the Japanese version of the OMSQ [23]. The OMSQ includes 12 items with multiple psychometric properties and sum scores range from 1 to 120 . Although there is no exact cut-off for poor prognosis in the Japanese population, a high sum score indicates risk of poor prognosis. Test-retest reliability (ICC = 0.92) has been established [23].

\subsubsection{The CSI}

We used the Japanese version of the CSI [24]. The CSI includes 25 items, with scores ranging from 0 to 4 . A higher total score indicates a greater deficit in nociplastic pain. The criterion-related validity, construct validity, and test-retest reliability $(\mathrm{ICC}=0.85)$ have been established [24].

\subsubsection{The PCS}

We used the Japanese version of the PCS [25] [26]. The PCS includes 13 items, with scores ranging from 0 to 4 . A higher total score indicates a greater deficit in pain catastrophizing. The construct validity and high internal consistency (Cronbach alpha of all items $=0.89$ ) have been established [26].

\subsubsection{The TSK}

We used the Japanese version of the TSK [27]. The TSK includes 17 items, with scores ranging from 1 to 4 . A higher total score indicates greater fear of move- 
ment. The concurrent validity and high internal consistency (Cronbach alpha of all items $=0.85)$ have been established [27].

\subsubsection{The HADS}

We used the Japanese version of the HADS [28]. The HADS includes 14 items, with scores ranging from 0 to 3 . Odd number items are for depression and even number items are for anxiety. A high total score indicates greater anxiety or depression. The construct validity has been established [28]. The participants rated the magnitudes of anxiety and depression by considering their status during the last one week.

\subsubsection{The PCSQ}

We used the Japanese version of the PCSQ [29], which includes two items; 1) how much control patients felt they had over pain, and 2) how much patients were able to decrease pain [30]. The scores range from 0 to $6(0=$ no control $/$ cannot decrease it at all, $3=$ some control $/$ can decrease it somewhat, and $6=$ complete control/can decrease it completely). A higher total score indicates greater confidence in a management strategy for pain. The construct validity has been established [29].

\subsubsection{The 11-Point GRCS}

We used the 11-point GRCS for perceived change immediately after the MDT physical evaluation, which was recommended as a standard measure for research on LBP [20]. The minimum clinically important change is 2 [20].

\subsection{Statistical Analysis}

Considering a feature of the pilot study, a minimum border for strong correlation of 0.8 was used for a priori sample size estimation. Consequently, 10 participants were estimated ( $\alpha=0.05$ and $\beta=0.20$ ) [31].

Descriptive analysis was used to summarize variables as the mean [standard deviation (SD)] and \% (number). Non-parametric analyses were used due to the small sample size. All statistical analyses were performed with SPSS (version 21.0, IBM Corporation, New York, USA). Statistical significance was set at $P<$ 0.05 .

The Spearman's $\rho$-values were calculated between the patients' and therapist's PROM scores immediately before and after the MDT physical evaluation for the following tests: 1) the PCS, 2) the TSK, 3) the HADS, and 4) the PCSQ. The following interpretation of the $\rho$-value was used: very weak $=0-0.19$, weak $=0.20$ 0.39 , moderate $=0.40-0.59$, strong $=0.60-0.79$, very strong $=0.80-1.0[32]$. The Wilcoxon signed-rank test was conducted for the patient's PROM scores given immediately before and after the MDT physical evaluation: 1) the PCS, 2) the TSK, 3) the HADS, and 4) the PCSQ, where the effect size r was also calculated. The following interpretation of the $r$-value was used: small effect $=0.1$, medium effect $=0.3$, and large effect $=0.5$ [33]. 


\section{Results}

The characteristics of the 10 participants are summarized in Table 1. Provisional MDT classification was Derangement Syndrome for 8 participants and Posture Syndrome for 2 participants. There were no missing data. None of the participants experienced any adverse event.

Table 2 presents $\rho$-values between patient and therapist PROM scores immediately before and after the MDT physical evaluation for: 1) the PCS, 2) the TSK, 3 ) the HADS, and 4) the PCSQ.

Table 3 presents the results of the Wilcoxon signed-rank test for the following patient measures taken immediately before and after the MDT physical evaluation: 1) the PCS, 2) the TSK, 3) HADS, and 4) the PCSQ. Statistically significant improvement with a large effect size was detected in the PCS, TSK, and PCSQ. Most participants (80\%) rated confidence to control the pain (response of $4-6$ ) and confidence to decrease the pain (response of $4-6$ ) higher after the MDT physical evaluation.

Table 1. Characteristics of the 10 participants.

\begin{tabular}{cc}
\hline Age (year) & $21.5(3.7)$ \\
\hline Gender (number of men), (\%) & $1(10)$ \\
Body Mass Index $\left(\mathrm{kg} / \mathrm{m}^{2}\right)$ & $21.7(1.6)$ \\
Symptom duration (months) & $18.5(20.7)$ \\
Symptom location $(1-6)$ & $2.4(1.3)$ \\
Oswestry Disability Index (\%) & $23.0(5.0)$ \\
P4 (0 - 40) & $20.2(7.6)$ \\
Central Sensitization Inventory $(0-100)$ & $50.0(7.9)$ \\
Global Recovery of Change Scale $5(-5-5)$ (number), (\%) & $28.0(11.8)$ \\
Global Recovery of Change Scale $4(-5-5)$ (number), (\%) & $3(30)$ \\
Global Recovery of Change Scale $3(-5-5)$ (number), (\%) & $5(50)$
\end{tabular}

Values are presented as mean (SD) unless otherwise specified.

Table 2. Spearman's $\rho$-values ( $P$-value) between the patients' and therapist's scores.

\begin{tabular}{ccc}
\hline Measure & Time 1 & Time 2 \\
\hline Pain Catastrophizing Scale & $0.65(0.043)$ & $0.81(0.005)$ \\
Tampa Scale for Kinesiophobia & $0.78(0.008)$ & $0.74(0.016)$ \\
Anxiety subscale of the Hospital Anxiety and Depression Scale & $0.34(0.332)$ & $0.35(0.316)$ \\
Depression subscale of the Hospital Anxiety and Depression Scale & $0.20(0.581)$ & $0.20(0.581)$ \\
Pain Coping Strategy Questionnaire & $-0.42(0.221)$ & $0(>0.999)$ \\
\hline
\end{tabular}

Time 1: Patients' scores before the Mechanical Diagnosis and Therapy (MDT) history taking vs physical therapist's scores before the MDT physical evaluation (but after the MDT history taking). Time 2: Patients' scores after the MDT physical evaluation vs physical therapist's scores after the MDT physical evaluation. 
Table 3. Wilcoxon signed-rank tests for the patients' measures immediately before the mechanical diagnosis and therapy (MDT) history taking and immediately after the MDT physical evaluation.

\begin{tabular}{ccccc}
\hline Measure & $\begin{array}{c}\text { Before the MDT } \\
\text { history taking }\end{array}$ & $\begin{array}{c}\text { After the MDT } \\
\text { physical evaluation }\end{array}$ & $P$-value & Effect size (r) \\
\hline Pain Catastrophizing Scale $(0-72)$ & $25.0(4.8)$ & $22.0(5.9)$ & 0.016 & 0.76 \\
Tampa Scale for Kinesiophobia $(17-68)$ & $21.7(5.1)$ & $19.9(6.0)$ & 0.027 & 0.72 \\
Anxiety subscale of the Hospital Anxiety and Depression Scale $(0-21)$ & $11.2(3.9)$ & $11.3(4.0)$ & 0.317 & 0.32 \\
Depression subscale of the Hospital Anxiety and Depression Scale $(0-21)$ & $13.7(2.9)$ & $13.7(2.9)$ & $>0.999$ & Not applicable \\
Pain Coping Strategy Questionnaire $(0-12)$ & $5.9(1.5)$ & $8.5(1.4)$ & 0.004 & 0.91 \\
\hline
\end{tabular}

Values are presented as mean $(\mathrm{SD})$.

\section{Discussion}

The current study preliminarily investigated whether a physical therapist trained through the MDT diploma program could guess patients' PROM scores through the MDT history taking and the MDT physical evaluation. Differently from the previous study [4], there were strong positive correlations between the patients' and therapist's scores in the PCS and the TSK. The results are based on one MDT therapist and generalizability of the findings is therefore limited. However, the results justify the need for further studies to explore effective post-graduate training to enhance synchronization of patient psychological status and therapist's guess. This is a prerequisite to developing a therapeutic alliance between the patient and therapist in order to provide a patient centered approach [34].

The strong positive correlations between patients' and therapist's scores in the PCS and TSK indicate important factors to consider when providing patients with MDT. First, clinical reasoning processes in the MDT physical evaluation utilize reactions of symptoms and physical functions to certain mechanical loadings. By choosing an appropriate mechanical loading from the beginning of the MDT physical evaluation, the therapist will avoid aggravating symptoms and decreasing motivation for further evaluations. Therefore, understanding the magnitude of pain catastrophizing and kinesiophobia is critical before the MDT physical evaluation. Second, therapists' fear avoidance increases support of patients' sick leave avoidance for returning to work and normal activity [35]. Thus, the discrepancy between physical therapists' guess for pain catastrophizing and kinesiophobia and the patients' perception should be minimized. The finding of a strong correlation between the patients' and therapist's scores in the PCS and TSK after the MDT physical evaluation indicates a minimized risk of the therapist's negative influence to the patient's prognosis.

The PCS and the TSK scores statistically improved after one MDT session. These findings indicate the possibility that pain catastrophizing and kinesiophobia have been adjusted through experiential learning by the patient about their response to pain in the mechanical loading examinations. It is also possible that as active listening and motivational interviewing are included in the MDT diploma program, the application of these skills combined with the mechanical 
loading examinations resulted in the improvement of PCS and TSK scores over only one MDT session. A systematic review including clinical trials of a certain duration demonstrated that MDT may improve fear avoidance belief [12], but dynamic psychological changes may also occur immediately though the MDT.

There was no strong correlation between the patients' and therapist's scores in the HADS, and the HADS scores did not change over the MDT session. One reason could be the psychometric property of the HADS. The lack of immediate change over one MDT session is not surprising because the participant rates their psychological status over the last week. The HADS was used in the current study because it was used in the previous study [4]. However, other measures than the HADS have been recommended for assessing anxiety and depression in individuals with musculoskeletal disorders [36]. A previous study [5] showing a correlation between patient's anxiety and therapist's rating $(\rho=0.86)$ used an 11-point NRS about anxiety in the 10-item Orebro Musculoskeletal Pain Screening Questionnaire [37]. Therefore, the current pilot study suggests to use other measures than the HADS such as the Pain Anxiety Symptoms Scale-20 [38] [39] for anxiety and the depression subscale Depression, Anxiety and Stress Scales Short Form [40] for depression in future studies.

Regarding the PCSQ scores, there was an improvement through the MDT session, and $80 \%$ of the participants gained confidence in their ability to control or decrease the pain. Indeed, all participants had improvements greater than the clinically meaningful important change of 2 in the GRCS. The results align with the previous study [11], indicating that the MDT results in positive behavioral change. Meanwhile, there was no correlation between the patients' and therapist's scores in the PCSQ. Although one reason may be the lack of confirmation of the psychometric property of the PCSQ, the finding may indicate a time requirement for patient's self-experiential learning about their response to pain and mechanical loading to reach a shared consensus in confidence of self-management between the patient and therapist, as suggested by a previous study [11].

\section{Limitations}

The present study has two potential limitations. The first limitation is a bias in the sample. The participants were young university students and biased toward females. The ODI $>13 \%$ was used to include participants who match to a clinical population, but more sampling variety is required for future studies. The second limitation is that the findings were obtained from a $60 \mathrm{~min}$ face-to-face session. When the MDT is undertaken in a shorter than normal time or via telerehabilitation, it is unknown if the same results can be obtained and generalizability of the findings is limited.

\section{Conclusion}

The current study found preliminary evidence that a physical therapist trained through the MDT diploma program could guess the PCS and TSK scores of pa- 
tients with LBP through MDT history taking and physical evaluation, which justifies further investigations. The current study also demonstrated preliminary evidence of immediate improvement of pain catastrophizing, kinesiophobia, and confidence in a management strategy for pain in patients with LBP over one MDT session.

\section{Conflicts of Interest}

The authors declare no conflicts of interest regarding the publication of this paper.

\section{References}

[1] Linton, S.J. (2000) A Review of Psychological Risk Factors in Back and Neck Pain. Spine (Phila Pa 1976), 25, 1148-1156. https://doi.org/10.1097/00007632-200005010-00017

[2] Kamper, S.J., Apeldoorn, A.T., Chiarotto, A., Smeets, R.J., Ostelo, R.W., et al. (2015) Multidisciplinary Biopsychosocial Rehabilitation for Chronic Low Back Pain: Cochrane Systematic Review and Meta-Analysis. BMJ, 350, h444. https://doi.org/10.1136/bmj.h444

[3] Main, C.J. and George, S.Z. (2011) Psychosocial Influences on Low Back Pain: Why Should You Care? Physical Therapy, 91, 609-613. https://doi.org/10.2522/ptj.2011.91.5.609

[4] Miki, T., Kondo, Y., Takebayashi, T. and Takasaki, H. (2020) Difference between Physical Therapist Estimation and Psychological Patient-Reported Outcome Measures in Patients with Low Back Pain. PLoS ONE, 15, e0227999. https://doi.org/10.1371/journal.pone.0227999

[5] Beales, D., Kendell, M., Chang, R.P., Hamso, M., Gregory, L., et al. (2016) Association between the 10 Item Orebro Musculoskeletal Pain Screening Questionnaire and Physiotherapists' Perception of the Contribution of Biopsychosocial Factors in $\mathrm{Pa}$ tients with Musculoskeletal Pain. Manual Therapy, 23, 48-55. https://doi.org/10.1016/j.math.2016.03.010

[6] McKenzie, R. and May, S. (2003) The Lumbar Spine: Mechanical Diagnosis and Therapy. 2nd Edition, Spinal Publications New Zealand Ltd., Waikenae.

[7] Foster, N.E., Thompson, K.A., Baxter, G.D. and Allen, J.M. (1999) Management of Nonspecific Low Back Pain by Physiotherapists in Britain and Ireland. A Descriptive Questionnaire of Current Clinical Practice. Spine (Phila Pa 1976), 24, 1332-1342. https://doi.org/10.1097/00007632-199907010-00011

[8] Ladeira, C.E., Samuel Cheng, M. and Hill, C.J. (2015) Physical Therapists' Treatment Choices for Non-Specific Low Back Pain in Florida: An Electronic Survey. The Journal of Manual \& Manipulative Therapy, 23, 109-118. https://doi.org/10.1179/2042618613Y.0000000065

[9] Battie, M.C., Cherkin, D.C., Dunn, R., Ciol, M.A. and Wheeler, K.J. (1994) Managing Low Back Pain: Attitudes and Treatment Preferences of Physical Therapists. Physical Therapy, 74, 219-226. https://doi.org/10.1093/ptj/74.3.219

[10] Davies, C., Nitz, A.J., Mattacola, C.G., Kitzman, P., Howell, D., et al. (2014) Practice Patterns When Treating Patients with Low Back Pain: A Survey of Physical Therapists. Physiotherapy: Theory and Practice, 30, 399-408. https://doi.org/10.3109/09593985.2013.877547 
[11] Takasaki, H. (2017) Mechanical Diagnosis and Therapy Enhances Attitude towards Self-Management in People with Musculoskeletal Disorders: A Preliminary Evidence with a Before-After Design. SAGE Open Medicine, 5, 2050312117740986. https://doi.org/10.1177/2050312117740986

[12] Kuhnow, A., Kuhnow, J., Ham, D. and Rosedale, R. (in press) The McKenzie Method and Its Association with Psychosocial Outcomes in Low Back Pain: A Systematic Review. Physiotherapy: Theory and Practice.

[13] Takasaki, H., Saiki, T. and Iwasada, Y. (2014) McKenzie Therapists Adhere More to Evidence-Based Guidelines and Have a More Biopsychosocial Perspective on the Management of Patients with Low Back Pain than General Physical Therapists in Japan. Open Journal of Therapy and Rehabilitation, 2, 173-181. https://doi.org/10.4236/ojtr.2014.24023

[14] Takasaki, H., Iwasada, Y. and May, S. (2015) Attitude towards the Use of Mechanical Diagnosis and Therapy and Reliability of Classification Extremity Problems by Credentialed Therapists. Journal of Chiropractic Medicine, 14, 32-38. https://doi.org/10.1016/j.jcm.2014.12.002

[15] Stanton, T.R., Latimer, J., Maher, C.G. and Hancock, M.J. (2010) How Do We Define the Condition "Recurrent Low Back Pain"? A Systematic Review. European Spine Journal, 19, 533-539. https://doi.org/10.1007/s00586-009-1214-3

[16] Stanton, T.R., Latimer, J., Maher, C.G. and Hancock, M. (2009) Definitions of Recurrence of an Episode of Low Back Pain: A Systematic Review. Spine (Phila Pa 1976), 34, E316-322. https://doi.org/10.1097/BRS.0b013e318198d073

[17] Fujiwara, A., Kobayashi, N., Saiki, K., Kitagawa, T., Tamai, K., et al. (2003) Association of the Japanese Orthopaedic Association Score with the Oswestry Disability Index, Roland-Morris Disability Questionnaire, and Short-Form 36. Spine (Phila Pa 1976), 28, 1601-1607. https://doi.org/10.1097/01.BRS.0000077510.95462.39

[18] Fairbank, J.C., Couper, J., Davies, J.B. and O'Brien, J.P. (1980) The Oswestry Low Back Pain Disability Questionnaire. Physiotherapy, 66, 271-273.

[19] Tonosu, J., Takeshita, K., Hara, N., Matsudaira, K., Kato, S., et al. (2012) The Normative Score and the Cut-Off Value of the Oswestry Disability Index (ODI). European Spine Journal, 21, 1596-1602. https://doi.org/10.1007/s00586-012-2173-7

[20] Kamper, S.J., Maher, C.G. and Mackay, G. (2009) Global Rating of Change Scales: A Review of Strengths and Weaknesses and Considerations for Design. The Journal of Manual \& Manipulative Therapy, 17, 163-170. https://doi.org/10.1179/jmt.2009.17.3.163

[21] Takasaki, H., Aoki, S. and May, S. (2018) No Increase in 6-Week Treatment Effect of Mechanical Diagnosis and Therapy with the Use of the LUMOback in People with Non-Acute Non-Specific Low Back Pain and a Directional Preference of Extension: A Pilot Randomized Controlled Trial. Physiotherapy, 104, 347-353. https://doi.org/10.1016/j.physio.2018.06.001

[22] Spadoni, G.F., Stratford, P.W., Solomon, P.E. and Wishart, L.R. (2004) The Evaluation of Change in Pain Intensity: A Comparison of the P4 and Single-Item Numeric Pain Rating Scales. Journal of Orthopaedic \& Sports Physical Therapy, 34, 187-193. https://doi.org/10.2519/jospt.2004.34.4.187

[23] Takasaki, H. and Gabel, C.P. (2017) Cross-Cultural Adaptation of the 12-Item Örebro Musculoskeletal Screening Questionnaire to Japanese (ÖMSQ-12-J), Reliability and Clinicians' Impressions for Practicality. The Journal of Physical Therapy Science, 29, 1409-1415. https://doi.org/10.1589/jpts.29.1409

[24] Tanaka, K., Nishigami, T., Mibu, A., Manfuku, M., Yono, S., et al. (2017) Validation 
of the Japanese Version of the Central Sensitization Inventory in Patients with Musculoskeletal Disorders. PLoS ONE, 12, e0188719. https://doi.org/10.1371/journal.pone.0188719

[25] Nishigami, T., Mibu, A., Tanaka, K., Yamashita, Y., Watanabe, A., et al. (2017) Psychometric Properties of the Japanese Version of Short Forms of the Pain Catastrophizing Scale in Participants with Musculoskeletal Pain: A Cross-Sectional Study. Journal of Orthopaedic Science, 22, 351-356.

https://doi.org/10.1016/j.jos.2016.11.015

[26] Matsuoka, H. and Sakano, Y. (2007) Assessment of Cognitive Aspect of Pain: Development, Reliability, and Validation of Japanese Version of Pain Catastrophizing Scale. Japanese Journal of Psychosomatic Medicine, 47, 95-102.

[27] Kikuchi, N., Matsudaira, K., Sawada, T. and Oka, H. (2015) Psychometric Properties of the Japanese Version of the Tampa Scale for Kinesiophobia (TSK-J) in Patients with Whiplash Neck Injury Pain and/or Low Back Pain. Journal of Orthopaedic Science, 20, 985-992. https://doi.org/10.1007/s00776-015-0751-3

[28] Matsudaira, T., Igarashi, H., Kikuchi, H., Kano, R., Mitoma, H., et al. (2009) Factor Structure of the Hospital Anxiety and Depression Scale in Japanese Psychiatric Outpatient and Student Populations. Health and Quality of Life Outcomes, 7, 42. https://doi.org/10.1186/1477-7525-7-42

[29] Keiko, O. and Satoshi, S. (2002) Pain Experiences and Coping Strategies. Women's Studies Forum, 16, 143-157.

[30] Rosenstiel, A.K. and Keefe, F.J. (1983) The Use of Coping Strategies in Chronic Low Back Pain Patients: Relationship to Patient Characteristics and Current Adjustment. Pain, 17, 33-44. https://doi.org/10.1016/0304-3959(83)90125-2

[31] Hulley, S., Cummings, S., Browner, W., Grady, D. and Newman, T. (2013) Designing Clinical Research: An Epidemiologic Approach. 4th Edition, Lippincott Williams \& Wilkins, Philadelphia.

[32] Campbell, M.J. and Swinscow, T.D.V. (2011) Statistics at Square One. 11th Edition, BMJ Books, London.

[33] Cohen, J. (1988) Statistical Power Analysis for the Behavioral Sciences. 2nd Edition, Routledge, New York.

[34] Hamovitch, E.K., Choy-Brown, M. and Stanhope, V. (2018) Person-Centered Care and the Therapeutic Alliance. Community Mental Health Journal, 54, 951-958. https://doi.org/10.1007/s10597-018-0295-Z

[35] Gardner, T., Refshauge, K., Smith, L., McAuley, J., Hubscher, M., et al. (2017) Physiotherapists' Beliefs and Attitudes Influence Clinical Practice in Chronic Low Back Pain: A Systematic Review of Quantitative and Qualitative Studies. Journal of Physiotherapy, 63, 132-143. https://doi.org/10.1016/j.jphys.2017.05.017

[36] Bijker, L., Sleijser-Koehorst, M.L.S., Coppieters, M.W., Cuijpers, P. and Scholten-Peeters, G.G.M. (2020) Preferred Self-Administered Questionnaires to Assess Depression, Anxiety and Somatization in People with Musculoskeletal Pain-A Modified Delphi Study. The Journal of Pain, 21, 409-417.

[37] Linton, S.J. and Hallden, K. (1998) Can We Screen for Problematic Back Pain? A Screening Questionnaire for Predicting Outcome in Acute and Subacute Back Pain. The Clinical Journal of Pain, 14, 209-215. https://doi.org/10.1097/00002508-199809000-00007

[38] Coons, M.J., Hadjistavropoulos, H.D. and Asmundson, G.J. (2004) Factor Structure and Psychometric Properties of the Pain Anxiety Symptoms Scale-20 in a Community Physiotherapy Clinic Sample. European Journal of Pain, 8, 511-516. 
https://doi.org/10.1016/j.ejpain.2003.11.018

[39] Abrams, M.P., Carleton, R.N. and Asmundson, G.J.G. (2007) An Exploration of the Psychometric Properties of the PASS-20 with a Nonclinical Sample. The Journal of Pain, 8, 879-886. https://doi.org/10.1016/j.jpain.2007.06.004

[40] Henry, J.D. and Crawford, J.R. (2005) The Short-Form Version of the Depression Anxiety Stress Scales (DASS-21): Construct Validity and Normative Data in a Large Non-Clinical Sample. British Journal of Clinical Psychology, 44, 227-239. https://doi.org/10.1348/014466505X29657 